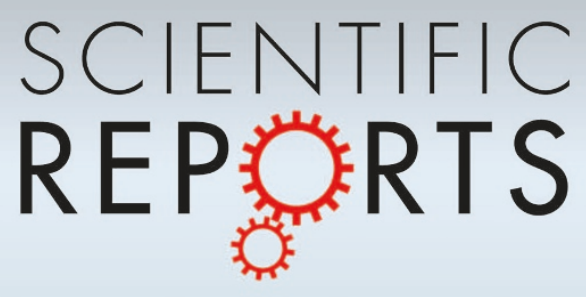

OPEN

SUBJECT AREAS:

NANOPARTICLES

METAL-ORGANIC

FRAMEWORKS

HOMOGENEOUS CATALYSIS

NANOCOMPOSITES

Received

10 May 2013

Accepted

25 June 2013

Published

18 July 2013

Correspondence and requests for materials should be addressed to

H.M. (maohui@ntu. edu.sg) or X.P.L.

(xpliao@scu.edu.cn)

\section{Biphasic catalysis using amphiphilic polyphenols-chelated noble metals as highly active and selective catalysts}

\author{
Hui Mao ${ }^{1,3}$, Hong $\mathrm{Yu}^{3}$, Jing Chen ${ }^{3} \&$ Xuepin Liao ${ }^{2}$
} 'College of Chemistry and Materials Science, Sichuan Normal University, Chengdu, PR China, $610068,{ }^{2}$ National Engineering
Laboratory for Clean Technology of Leather Manufacture, Sichuan University, Chengdu, PR China, 610065, ${ }^{3}$ School of Materials
Science and Engineering, Nanyang Technological University, 50 Nanyang Avenue, Singapore 639798.

In the field of catalysis, it is highly desired to develop novel catalysts that combine the advantages of both homogeneous and heterogeneous catalysts. Here we disclose that the use of plant pholyphenol as amphiphilic large molecule ligand/stabilizer allows for the preparation of noble metal complex and noble metal nanoparticle catalysts. These catalysts are found to be highly selective and active in aqueous-organic biphasic catalysis of cinnamaldehyde and quinoline, and can be reused at least 3 times without significant loss of activity. Moreover, the catalytic activity and reusability of the catalysts can be rationally controlled by simply adjusting the content of polyphenols in the catalysts. Our strategy may be extended to design a wide range of aqueous-organic biphasic catalysis system.

$\mathrm{T}$ o date, more than $90 \%$ of the industrial processes are relied on catalysis, such as chemical, pharmaceutics, materials, polymers and energy. The development of new catalysts has been the most important issue in catalysis science. Conventionally, the catalysts are classified into two typical categories, the homogeneous and heterogeneous catalysts ${ }^{1-3}$. Homogeneous catalysts are usually featured by high activity and high selectivity since homogeneous catalysts stay in the same phase as the reactants but the recovery of homogeneous catalysts from reaction media is extremely difficult and often requires tedious procedures ${ }^{4,5}$. Although heterogeneous catalysts provide convenient recovery of catalyst, the heterogenization of catalytic active species onto supporting matrices inevitably leads to loss of catalytic activity and/or selectivity owing to the strong interactions between supporting matrices and the catalytic active sites as well as the corresponding steric restriction ${ }^{6-8}$. Obviously, there is an acute need for developing new catalyst material that combines the advantages of both homogeneous (high activity and high selectivity) and heterogeneous catalysts (easy recovery and reusability).

In recent years, the concept of biphasic catalysis has attracted considerable attention and has emerged as promising strategy for developing catalysts with high activity and reusability ${ }^{9-11}$. In biphasic catalysis system, the catalyst resides in aqueous phase and the substrate resides in the other phase, immiscible organic phase. Upon reactions, the two phases are vigorously stirred to allow interfacial interactions between aqueous phase (catalyst) and organic phase (substrate). Once the reaction is completed, the two phases separate by simple decantation, where the aqueous phase contains the catalyst and organic phase contains the product. In principle, the aqueous catalyst can be conveniently cycled many times with high catalytic turnover number (TON). However, it has proven to be very challenging to prevent the leaching of metal catalyst from aqueous phase to organic phase because the conventionally used ligands are small hydrophilic molecules that have limited stabilizing effect towards metal species, such as sulfonated phosphines ${ }^{12-14}$. TPPTS $\left[\mathrm{P}\left(\mathrm{m}-\mathrm{C}_{6} \mathrm{H}_{4} \mathrm{SO}_{3} \mathrm{Na}\right)_{3}\right]$ is one of the most commonly used sulfonated phosphine ligands, which is chelated with metal ions by phosphine and provides the catalyst hydrophilicity by sulfonic groups. However, TPPTS belongs to monodentate ligands with limited stabilizing ability to chelate metal ions, and the activity of TPPTS-chelated metal catalyst is usually found to substantially decrease during cycling because of the leaching of metal species into organic phase. Although some bidentate sulfonated phosphines have been synthesized in order to increase the chelating ability of this type of ligand, these synthetic ligands require complicated synthesis route, and often involve toxic solvent ${ }^{15,16}$. Another challenging in developing biphasic system is to improve the mass transfer kinetics between two phases. The use of surfactant and phase transfer agent can substantially decrease the interfacial resistances while catalyst recycling would be quite difficult due to the ineffective phase separation caused by the use of surfactants ${ }^{17,18}$. One possible solution to solve both of the above problems is to prepare aqueous catalysts using amphiphilic large molecules as 


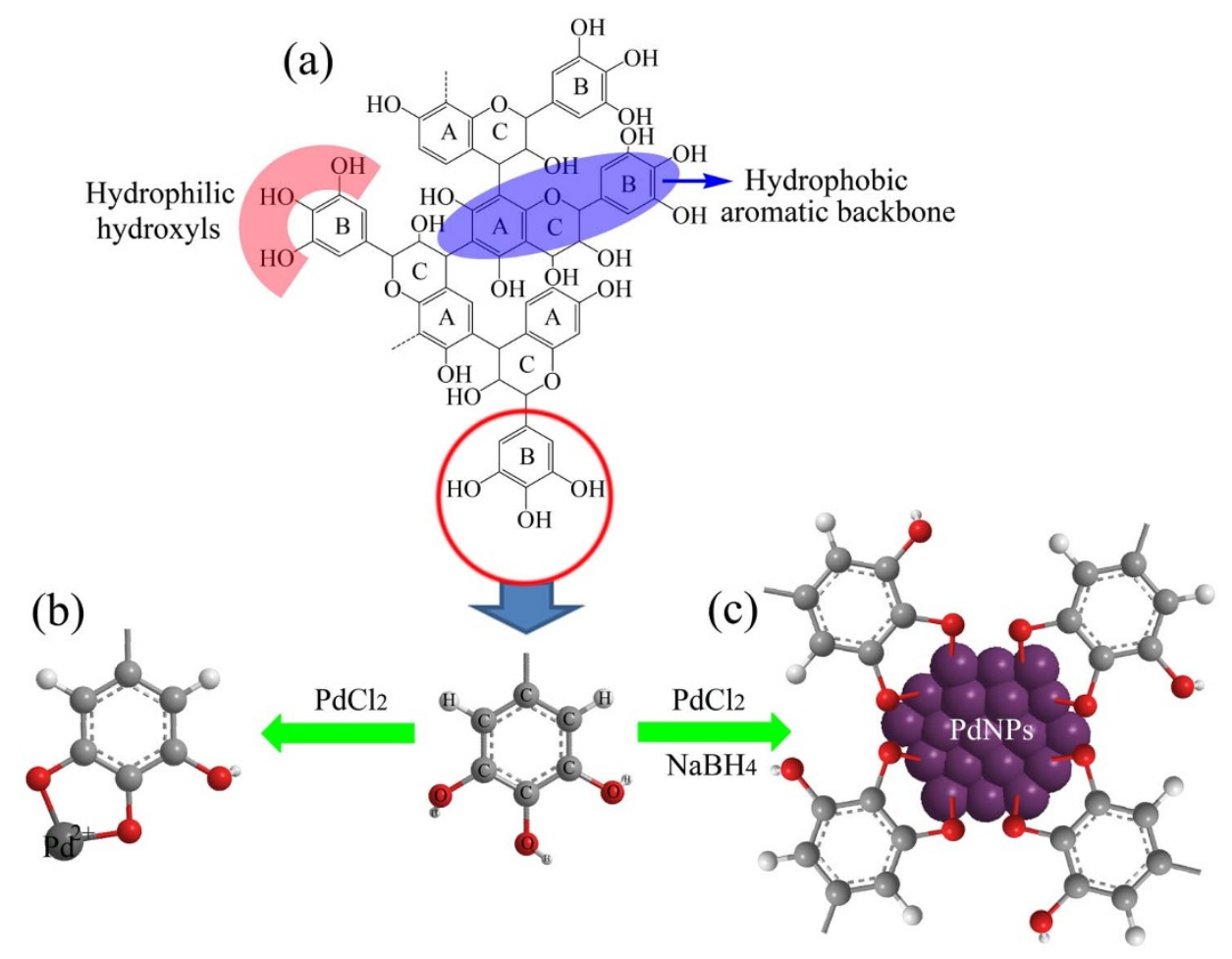

Figure 1 | Schematic illustration of preparation of polyphenols-chelated metal catalysts.

the ligands. In principle, the use of large molecule as ligand can exhibit stronger stabilizing effect to the metal species compared to the small molecule ligands, and the amphiphilic nature of ligands can effectively decrease the interfacial resistances while without suffering the problems with phase separation ${ }^{19-21}$. In this way, the as-designed catalysts can therefore exhibit high catalytic activity and good reusability in biphasic catalysis.

Here we attempt to use natural polymers as the ligands to replace the synthetic counterparts in order to provide a more environmentally benign and cost-effective methodology. Black wattle tannin (BWT), a kind of typical natural polyphenols extracted from plants, is used as bidentate ligand to chelate with metal ions. BWT is a mixture of poly-prodelphinidin with average molecular weight of 1250 , and contains a large number of ortho-phenolic hydroxyls at its $\mathrm{B}$ rings, which provide BWT very excellent chelating ability and good water-solubility. The macromolecular backbone of BWT is composed of hydrophobic aromatic rings, which can interact with the hydrophobic substrate and/or organic phase by hydrophobic interactions ${ }^{22,23}$. In this way, the interfacial resistances in biphasic catalysis can be substantially decreased by using BWT as amphiphilic ligand. In the present investigation, we first prepared highly active and recyclable noble metal complex (BWT- $\mathrm{Rh}^{3+}$ and $\mathrm{BWT}-\mathrm{Pd}^{2+}$ ) and noble metal nanoparticle (BWT-PdNPs and BWT-RhNPs) catalysts by using polyphenols as amphiphilic large molecule ligands. Subsequently, the catalytic properties of the catalysts were evaluated in aqueous-organic biphasic hydrogenation of cinnamaldehyde and quinoline, and the influences of experimental parameters on catalytic properties were also systematically investigated, such as polyphenol content, organic solvent, temperature and $\mathrm{H}_{2}$ pressure.

\section{Results}

A series of homogeneous BWT-Pd ${ }^{2+}$ complex catalysts were prepared by simply mixing different amount of BWT with $\mathrm{PdCl}_{2}$ aqueous solution, and the corresponding BWT-PdNPs catalysts were

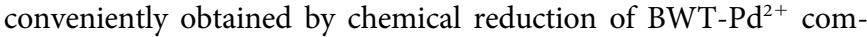
plex at room temperature using $\mathrm{NaBH}_{4}$ as reductant (see detail in experiments section). Fig. 1(b) illustrates the chelating interactions between BWT and $\mathrm{Pd}^{2+}$ as well as the stabilizing mechanism of BWT towards PdNPs respectively. For the chelating reaction, two adjacent phenolic hydroxyls of BWT at B rings are first dissociated by releasing the protons, and then act as bidentate ligands to chelate with $\mathrm{Pd}^{2+}$, giving the formation of highly stable five-membered chelating ring. Based on XPS analysis, the O 1s XPS spectrum of BWT shows a major peak of phenolic oxygens at $531.8 \mathrm{eV}$ while this peak is shifted to higher binding energy of $532.2 \mathrm{eV}$ after chelating with BWT (Supplementary Fig. S1), suggesting the donation of electrons from phenolic hydroxyls of BWT to $\mathrm{Pd}^{2+}$. As for the BWT-PdNPs, the stabilizing effects of BWT to PdNPs are confirmed by FTIR spectroscopy analysis (Supplementary Fig. S2). Compared with pure BWT, the stretching vibration peak of phenolic hydroxyls is narrowed at $3400 \mathrm{~cm}^{-1}$ after stabilized with PdNPs, and the peaks belong to aromatic rings of BWT also become weak in the vicinity of 1614$1458 \mathrm{~cm}^{-1}$. These differences suggest that PdNPs are mainly stabilized by the phenolic hydroxyls of BWT. Based on TEM observation, the as-prepared BWT-PdNPs gel solution contains very small Pd nanoparticles with diameter of $2 \sim 3 \mathrm{~nm}$ (Supplementary Fig. S3).
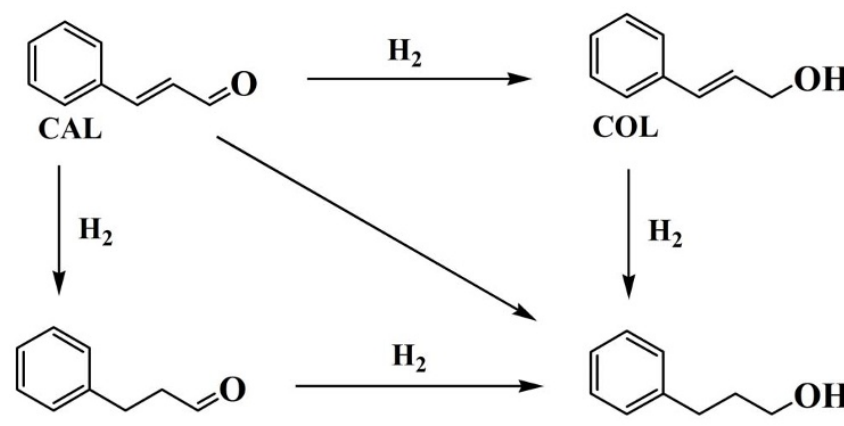

HCAL

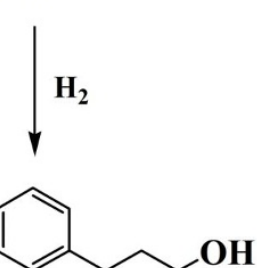

HCOL 
Hydrogenation of cinnamaldehyde is employed as the probe reaction to evaluate the catalytic activity, selectivity and reusability of the as-prepared aqueous catalysts. Since cinnamaldehyde is $\alpha$ - $\beta$ unsaturated aldehyde that contains two unsaturated bonds in molecule, the hydrogenation of cinnamaldehyde can generate different products. Fig. 2 illustrates the reaction pathways in hydrogenation of cinnamaldehyde. The hydrogenation of cinnamaldehyde in $\mathrm{C}=\mathrm{C}$ bond can form hydrocinnamaldehyde (HCAL) while the hydrogenation of cinnamaldehyde in $\mathrm{C}=\mathrm{O}$ bond leads to the formation of cinnamyl alcohol (COL). Further hydrogenation of these two intermediates results in the formation of hydrocinnamyl alcohol (HCOL). Although the hydrogenation of $\mathrm{C}=\mathrm{C}$ bond in $\alpha-\beta$ unsaturated aldehydes is both kinetically and thermodynamically more favorable than that of the $\mathrm{C}=\mathrm{O}$ group ${ }^{24}$, the $100 \%$ selective hydrogenation of CAL to HCAL is quite difficult.

Much to our delight, the selectivity of our BWT-Pd ${ }^{2+}$ and BWTPdNPs catalysts both exhibit $100 \%$ of selectivity to HCAL, as shown in Table 1. As we know, the adsorption of substrate on the catalyst will significantly influence the catalytic reaction. We speculate that due to the strengthened hydrophobic interactions between aromatic backbone of BWT and aromatic rings of CAL, the preferred hydrogenation of $\mathrm{C}=\mathrm{C}$ bond over $\mathrm{C}=\mathrm{O}$ bond will be promoted due to its close distance with the aromatic ring of CAL. This effect accompanied with the intrinsic high reactivity of $\mathrm{C}=\mathrm{C}$ hydrogenation, thus resulting in $100 \%$ selective hydrogenation of CAL to HCAL. We then screen the optimal conditions for CAL hydrogenation. The influence of solvent on catalytic activity is first investigated. As shown in Table 1 (Entry 1-3), when cyclohexane is used as the organic phase, the conversion yield of CAL is around $32.7 \%$ for BWT- $\mathrm{Pd}^{2+}$. By replace the organic phase to toluene and n-butanol, the corresponding conversion yields under the same experimental conditions are increased to $53.1 \%$ and $76.2 \%$, respectively. It is likely that the use of organic solvent with higher polarization is more beneficial for the biphasic reaction to proceed. Such phenomenon is also confirmed when using BWT-PdNPs catalyst, where the corresponding conversion yields are $51.3 \%, 75.5 \%$ and $91.2 \%$, respectively, when using cyclohexane, toluene and n-butanol as the organic phase, respectively. For both of our catalysts, the amphiphilic BWT molecules can not only interact with hydrophobic substrates by hydrophobic interactions but also exhibit affinity to polarized molecules by multiple hydrogen-bonding interactions. When the organic phase with stronger polarization is employed, the phenolic hydroxyls of BWT may form hydrogen bond with the polarized partition of organic solvent like the hydroxyl oxygen of n-butanol, which further increase the contact chance of catalyst to the substrate dissolved in the organic solvent. Catalytic reaction carried out at high pressure may lead to deep hydrogenation of CAL to HCOL. To reveal that both of our catalysts are highly selective to HCAL, the biphasic reaction is conducted at even higher $\mathrm{H}_{2}$ pressure of 3.0 and 4.0 MPa. In Table 1, entry $4-5$, the BWT-Pd ${ }^{2+}$ and BWT-PdNPs catalysts still exhibit substantially increased conversion yield of substrate due to the increase of dissolved $\mathrm{H}_{2}$ in solvent at high pressure. However, no other product, such as HCOL, is detected, and the selectivity to HCAL is still $100 \%$. These facts suggest that the selectivity of our catalysts should be mainly derived from their intrinsic thermodynamically properties rather than the controlling of catalytic parameters. In Table 1 Entry 6-11, the increase of reaction temperature from 303-363K promotes the activity of both catalysts. The conversion yield of substrate over BWT-Pd ${ }^{2+}$ is increased from $3.2 \%$ to $77.3 \%$, and that over BWT-PdNPs is increased from $9.1 \%$ to $93.5 \%$. The influence of BWT content on the catalytic activity of the catalysts is also investigated in detail. As shown in Table 1, Entry 11-15, the increase of BWT content causes an obvious decrease of activity, where the conversion yield of substrate is decreased from $91.3 \%$ to $3.3 \%$ when the dosage of BWT in the BWT- $\mathrm{Pd}^{2+}$ catalyst is changed from 2 to $60 \mathrm{mg}$. Similar tendency is also observed for BWT-PdNPs catalyst. The corresponding conversion yield of substrate is decreased from $95.7 \%$ to $10.1 \%$ when the dosage of BWT in the BWT-PdNPs is increased from 2 to $60 \mathrm{mg}$. According to the literatures $^{25,26}$, when ligands are chelated with metal species or stabilize with metal nanoparticles, the molecular structure of ligands will inevitably cause some extent of steric hindrance, and such influence is found to be more severe for those ligands with large molecular structure. As for BWT, it contains a large rigid aromatic backbone that causes steric hindrance to the catalysts. At high content of BWT, such hindrance effect can be intensively strengthened via the formation of hydrogen bonds and/or hydrophobic bonds among BWT molecules, and a dense supramolecular shell may be formed around the active metal species, which restrict the access of substrates to catalytic active sites, resulting in loss of activity as observed in Table 1, Entry 11-15. Although the increase of BWT content leads to the loss of catalyst activity, it can increase the cycling stability of the catalyst.

As shown in Table 2, for both BWT-Pd ${ }^{2+}$ and BWT-PdNPs catalysts, their reusability is improved along with the increase of BWT content. For the hydrogenation of CAL over BWT-Pd ${ }^{2+}$, as an example, when the content of BWT is $5 \mathrm{mg}$ in the catalyst, the conversion yield of substrate is decreased from $85.7 \%$ to $54.3 \%$ after cycled from 1-run to 5-run. By increasing the BWT content to $15 \mathrm{mg}$, the conversion yield of substrate in 5-run (73.6\%) is still comparable

Table 1 | Condition screening* in aqueous-organic biphasic hydrogenation of cinnamaldehyde using BWT-Pd ${ }^{2+}$ and BWT-PdNPs catalysts

\begin{tabular}{|c|c|c|c|c|c|c|c|c|}
\hline Entry & Solvent & Temp. (K) & Pressure (MPa) & BWT (mg) & Product & Selectivity (\%) & $\begin{array}{l}\text { TOF of BWT-Pd }{ }^{2+} \\
\left(\mathrm{mol} \cdot \mathrm{mol}^{-1} \cdot \mathrm{h}^{-1}\right)\end{array}$ & 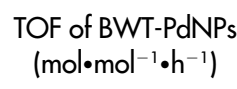 \\
\hline 1 & Cyclohexane & 353 & 2.0 & 15.0 & $\mathrm{HCAL}$ & 100 & 81.75 & 128.25 \\
\hline 2 & Tolvene & 353 & 2.0 & 15.0 & $\mathrm{HCAL}$ & 100 & 132.75 & 188.75 \\
\hline 4 & n-butanol & 353 & 3.0 & 15.0 & $\mathrm{HCAL}$ & 100 & 198.5 & 233.75 \\
\hline 5 & n-butanol & 353 & 4.0 & 15.0 & $\mathrm{HCAL}$ & 100 & 203.25 & 238.75 \\
\hline 6 & n-butanol & 303 & 2.0 & 15.0 & $\mathrm{HCAL}$ & 100 & 8 & 22.75 \\
\hline 9 & n-butanol & 333 & 2.0 & 15.0 & $\mathrm{HCAL}$ & 100 & 85.25 & 113.25 \\
\hline 10 & n-butanol & 343 & 2.0 & 15.0 & $\mathrm{HCAL}$ & 100 & 129.25 & 159.5 \\
\hline 11 & n-butanol & 363 & 2.0 & 15.0 & $\mathrm{HCAL}$ & 100 & 193.25 & 233.75 \\
\hline 12 & n-butanol & 353 & 2.0 & 2.0 & $\mathrm{HCAL}$ & 100 & 228.25 & 239.25 \\
\hline 13 & n-butanol & 353 & 2.0 & 5.0 & $\mathrm{HCAL}$ & 100 & 214.25 & 232.75 \\
\hline 14 & n-butanol & 353 & 2.0 & 30.0 & $\mathrm{HCAL}$ & 100 & 75.25 & 143.25 \\
\hline
\end{tabular}




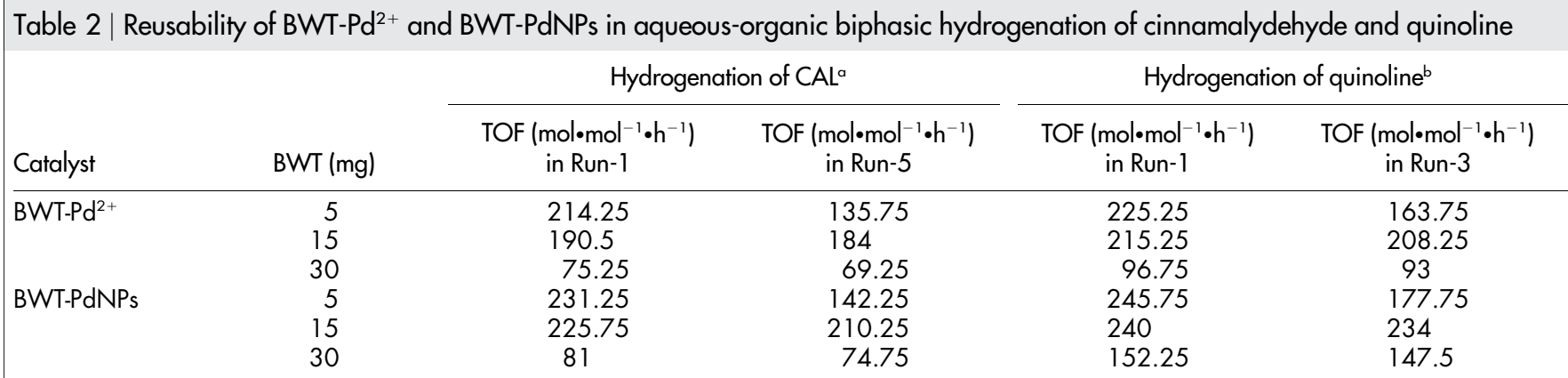

aPd: $10.0 \mu \mathrm{mol}$, Cinnamalydehyde: $1.25 \mathrm{mmol}$, Solvent: n-butanol, Temperature: $353 \mathrm{~K}$, Pressure: $2.0 \mathrm{MPa}$, Reaction time: $30 \mathrm{~min}, 100 \%$ selectivity to HCAL.

bPd: $50.0 \mu \mathrm{mol}$, Quinoline: $5 \mathrm{mmol}$, Solvent: n-butanol, Temperature: $353 \mathrm{~K}$, Pressure: $2.0 \mathrm{MPa}$, Reaction time: $30 \mathrm{~min}, 100 \%$ selectivity to py-THQ.

to that in 1-run $(76.2 \%)$. Further increasing the BWT content to $30 \mathrm{mg}$, the catalyst also shows quite well cycling stability. Similar phenomenon is still observed for the BWT-PdNPs catalyst. To further confirm such influence of BWT to the reusability of the catalyst. The biphasic hydrogenation of quinoline is conducted over BWT$\mathrm{Pd}^{2+}$ and BWT-PdNPs catalysts, respectively. Similarly, the low content of BWT in catalyst can result in quite high catalytic activity in the 1-run but a substantial decrease of conversion yield is observed in the following cycles. By increasing the content of BWT to a proper amount, such as $15 \mathrm{mg}$, appreciable high activity and satisfied reusability can be achieved. It should also be noted that 1,2,3,4-tetrahydroquinoline (py-THQ) is the only catalytic product of quinoline, and there is no 5,6,7,8-tetrahydroquinoline (bz-THQ) or decahydroquinoline (DHQ) detected in the reaction. Compared with most of the previous researches, our catalysts show obvious advantages both in activity and reusability. For example, Melean and coworkers applied hydrosoluble complexes $[\mathrm{Rh}(\mathrm{CO})(\mathrm{Pz})(\mathrm{TPPTS})]_{2}$ (TPPTS $=$ triphenylphosphine-trisulfonated) in biphasic hydrogenation of cinnamaldehyde ${ }^{27}$. When the biphasic reaction was conducted at $343 \mathrm{~K}$ for $24 \mathrm{~h}$ using cetyl-trimethylammonium chloride as the phase transfer agent, the conversion yield of cinnamaldehyde is only $55 \%$ in the 1 -run, and the conversion yield shows an obvious decrease during cycling. In the 5-run, conversion yield of cinnamaldehyde is as low as $\sim 40 \%$.

In principle, our strategy for the synthesis of $\mathrm{BWT}-\mathrm{Pd}^{2+}$ and BWT-PdNPs catalysts can extend to synthesize other noble metal catalyst because BWT has strong chelating ability towards most of the transition metal ions with d-orbits ${ }^{28,29}$. To demonstrate this, we prepared BWT- $\mathrm{Rh}^{3+}$ and BWT-RhNPs catalysts. The chelating interactions between BWT and $\mathrm{Rh}^{3+}$ are first confirmed by UV-vis (Supplementary Fig. S4) and HNMR analyses (Supplementary Fig. S5), and the formation of RhNPs is verified by TEM image (Supplementary Fig. S6). Subsequently, the BWT-Rh ${ }^{3+}$ and BWTRhNPs are employed as catalysts in biphasic hydrogenation of CAL. As shown in Table 3, the BWT- $\mathrm{Rh}^{3+}$ complexes exhibit high activity and selectivity in biphasic hydrogenation of CAL. HCAL is the only product with $100 \%$ selectivity. For BWT- $\mathrm{Rh}^{3+}$ catalyst, its average turnover frequency (TOF) value is as high as $471.0 \mathrm{~mol} \cdot \mathrm{mol}^{-1} \cdot \mathrm{h}^{-1}$ in the 1 -run. In the 2-run, the average TOF is still high up to $459.0 \mathrm{~mol} \cdot \mathrm{mol}^{-1} \cdot \mathrm{h}^{-1}$. In the 5 -run, the average TOF is $435.0 \mathrm{~mol} \cdot$ $\mathrm{mol}^{-1} \cdot \mathrm{h}^{-1}$, retaining $92.36 \%$ catalytic activity of the 1 -run. For biphasic hydrogenation of quinoline over BWT-RhNPs catalyst, the average TOF in the 1 -run is $163 \mathrm{~mol} \cdot \mathrm{mol}^{-1} \cdot \mathrm{h}^{-1}$, and this value is still kept at $157 \mathrm{~mol} \cdot \mathrm{mol}^{-1} \cdot \mathrm{h}^{-1}$ in the 3-run, also showing no significant loss of activity during cycling. We also performed biphasic hydrogenation of quinoline over commercial $\mathrm{RhCl}(\mathrm{CO})(\mathrm{TPPTS})_{2}$ catalyst purchased from Sigma-Aldrich cooperation. When the biphasic hydrogenation was conducted at $363 \mathrm{~K}$, the TOF of $\mathrm{RhCl}(\mathrm{CO})(\mathrm{TPPTS})_{2}$ is $92.6 \mathrm{~mol} \cdot \mathrm{mol}^{-1} \cdot \mathrm{h}^{-1}$, only counting for $56.81 \%$ TOF of the BWT-RhNPs catalyst.

\section{Discussion}

In conclusion, we have reported a systematic study, in which plant polyphenols were used as amphiphilic large molecule ligands to synthesize a class of complex and nanoparticle catalysts. The orthophenolic hydroxyls of polyphenols are chelated with transition metal ions to form noble metal complexes catalysts. The rigid aromatic backbone of polyphenols provides steric hindrance to metal nanoparticles, ensuring good stability. More importantly, the amphiphilic nature of polyphenols affords the as-prepared catalysts high activity and reusability in biphasic hydrogenation reactions, and the catalytic activity and reusability of the catalysts can be rationally controlled by simply adjusting the content of polyphenols in the catalysts. Our further work will focus on the synthesis of polyphenols-stabilized bimetallic nanocatalysts, and their applications in biphasic catalytic reactions.

\section{Methods}

Preparation of BWT-Pd ${ }^{2+}$ and BWT-PdNPs catalysts. $10 \mu \mathrm{mol}$ (or $50 \mu \mathrm{mol}$ ) $\mathrm{Pd}^{2+}$ was dissolved into $3.0 \mathrm{~mL}$ of deionized water, and then different amounts of BWT ( $2 \mathrm{mg}, 5 \mathrm{mg}, 15 \mathrm{mg}, 30 \mathrm{mg}$ and $60 \mathrm{mg}$ ) were added to the $\mathrm{Pd}^{2+}$ solution, followed by constant stirring for $20 \mathrm{~min}$. The resultant solution is BWT-Pd ${ }^{2+}$ complexes catalyst. BWT-PdNPs catalysts were prepared by reducing the above BWT-Pd ${ }^{2+}$ complexes catalysts using $5.0 \mathrm{~mL}$ of $0.010 \mathrm{~g} \mathrm{NaBH}_{4}$ aqueous solution.

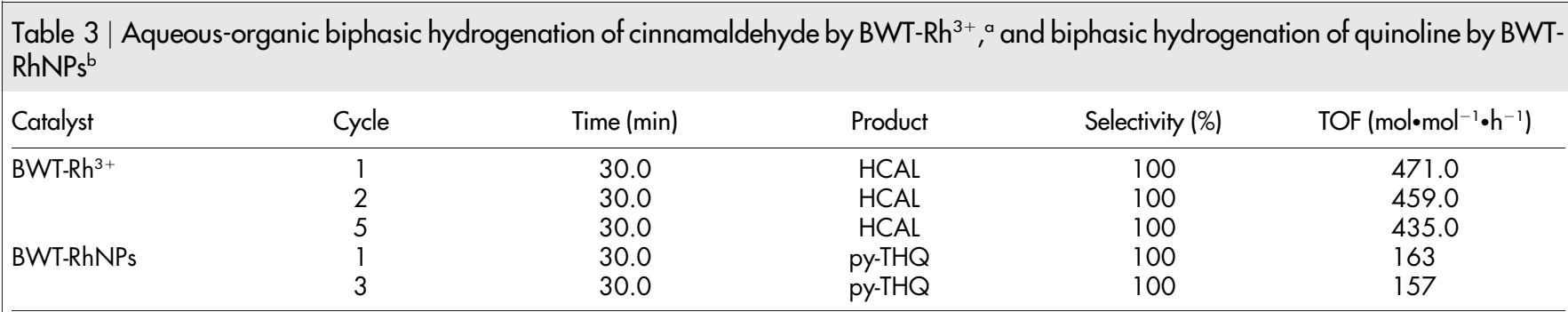


Preparation of BWT-Rh ${ }^{3+}$ and BWT-RhNPs catalysts. $5 \mu \mathrm{mol} \mathrm{Rh^{3+ }}$ was dissolved into $3.0 \mathrm{~mL}$ of deionized water, and then $15 \mathrm{mg}$ of BWT was added to the $\mathrm{Rh}^{3+}$ solution, followed by constant stirring for $20 \mathrm{~min}$. The resultant solution is BWT$\mathrm{Rh}^{3+}$ complexes catalyst. BWT- RhNPs catalysts were prepared by reducing the above BWT-Rh ${ }^{3+}$ complexes catalysts using $5.0 \mathrm{~mL}$ of $0.010 \mathrm{~g} \mathrm{NaBH}_{4}$ aqueous solution.

Characterization and biphasic hydrogenation. X-ray Photoelectron Spectroscopy (XPS) of BWT and BWT-Pd were carried out using a VG Scientific ESCALAB 200 instrument. The morphology of nanoparticles were determined using Transmission Electron Microscopy (TEM, Tecnai G2 F20 S-TWIN, U.S.) operated at an acceleration voltage of $200 \mathrm{kV}$. Fourier Transform Infrared spectoscopy (FT-IR) of BWT and BWT-Pd were tested by Nicolet 6700 instrument. The UV-vis spectra of BWT and BWT-Rh ${ }^{3+}$ were measured by UV-3600 UV-vis spectrophotometer (Shimadzu.Japan). Proton nuclear magnetic resonance (HNMR) was measured by Bruker DPX400 NMR instrument using DMSO- $\mathrm{d}_{6}$ as solvent.

Biphasic hydrogenation was conducted by adding desired amount of catalyst solution and substrate dissolved in organic solvent (or just substrate) into a stainless steel reactor. After the reactor was flushed three times with $\mathrm{H}_{2}$, catalytic hydrogenation was conducted under constant $\mathrm{H}_{2}$ pressure and temperature. When the reaction was completed, the organic phase was collected by decantation and analyzed by gas chromatography (GC) while the aqueous phase was then reused. The turnover frequency (TOF) of the catalyst in the hydrogenation experiments was calculated using the following equation:

TOF $\left(\mathrm{mol} \cdot \mathrm{mol}^{-1} \cdot \mathrm{h}^{-1}\right)=$ Hydrogenated substrate $(\mathrm{mol}) /$

[Noble metal in catalyst $(\mathrm{mol}) \times \mathrm{t}(\mathrm{h})$ ]

1. Noritaka, M. \& Makoto, M. Heterogeneous catalysis. Chem. Rev. 98, 199-218 (1998).

2. Corma, A., García, H. \& Llabrés, F. X. Engineering Metal Organic Frameworks for Heterogeneous Catalysis. Chem. Rev. 110, 4606-4655 (2010).

3. David, J. C. Homogeneous catalysis--new approaches to catalyst separation, recovery, and recycling. Science 299, 1702-1706 (2003).

4. Bergbreiter, D. E., Tian, J. \& Hongfa, C. Using soluble polymer supports to facilitate homogeneous catalysis. Chem Rev. 109, 53-82 (2009).

5. Kathrin, J., Kristin, S. \& Matthias, B. Homogeneous catalysis using iron complexes: recent developments in selective reductions. Chem. Commun. $\mathbf{4 7}$ 4849-4859 (2011).

6. Weiting, Y., Marc, D. P. \& Jingguang, G. C. Review of Pt-Based Bimetallic Catalysis: From Model Surfaces to Supported Catalysts. Chem. Rev. 112, 5780-5817 (2012)

7. Sankaranarayanapillai, S., Volker, S. \& Werner, R. Magnetically separable nanocatalysts: bridges between Homogeneous and Heterogeneous Catalysis. Angew. Chem. Int. Ed. 49, 3428-3459 (2010).

8. Herney-Ramireza, J., Miguel, A. V. \& Luis, M. M. Heterogeneous photo-Fenton oxidation with pillared clay-based catalysts for wastewater treatment: a review. Appl. Catal. B 98, 10-26 (2010).

9. Cornils, B. Industrial aqueous biphasic catalysis: status and directions. Org. Process Res. Dev. 2, 121-127 (1998).

10. Fabian, J., Jean-Michel, A. \& Alfons, B. Ionic liquids and dense carbon dioxide: a beneficial biphasic system for catalysis. Chem. Rev. 111, 322-353 (2011).

11. Christian, W. K., Richard, W. F. \& Cornils B. Aqueous biphasic catalysis: buhrchemie/rhone-poulenc oxo process. Appl. Catal. A 221, 219-225 (2001).

12. Nathalie, P. \& Duncan, W. B. Homogeneous catalysts based on water-soluble phosphines. Coord. Chem. Rev. 241, 1-25 (2003).

13. Cornils, B. \& Emile, G. K. Introducing TPPTS and related ligands for industrial biphasic processes. J. Organomet. Chem. 502, 177-186 (1995).

14. Kevin, H. S. Hydrophilic ligands and their application in aqueous-phase metalcatalyzed reactions. Chem. Rev. 109, 643-710 (2009).

15. Kotzabasakis, V., Georgopoulou, E., Pitsikalis, M., Georgopoulou, E. \& Pitsikalis, M. Catalytic conversions in aqueous media: a novel and efficient hydrogenation of polybutadiene-1,4-block-poly(ethylene oxide) catalyzed by Rh/TPPTS complexes in mixed micellar nanoreactors. J. Mol. Catal. A Chem. 231, 93-101 (2005).
16. Neatu, F. et al. Hydrotalcite docked Rh-TPPTS complexes as efficient catalysts for the arylation of 2-cyclohexen-1-one in neat water. Catal Today 139, 161-167 (2008).

17. Anders, R. \& Brian, E. H. CTAB micelles and the hydroformylation of octene with rhodium/TPPTS catalysts: evidence for the interaction of TPPTS with micelle surfaces. J. Mol. Catal. A: Chem. 189, 195-202 (2002).

18. Asif, A. D., Dharmesh, U. P., Hari, C. B. \& Raksh, V. J. $\mathrm{CoCl}_{2}$ (TPPTS) $)_{2}$ catalyzed hydroformylation of 1-octene and 1-decene in the presence of surfactant and co-solvents in a biphasic medium. J. Mol. Catal. A: Chem. 282, 99-106 (2008).

19. Marcel, S. G. et al. Accelerated Biphasic Hydroformylation by vesicle formation of amphiphilic diphosphines. J. Am. Chem. Soc. 122, 1650-1657 (2000).

20. Hicham, A., Krystyna, B., Stephanie, C. \& Chantal, L. Thermoregulated aqueous biphasic catalysis of Heck reactions using an amphiphilic dipyridyl-based ligand. Green Chem. 10, 1197-1203 (2008).

21. Natacha, S. et al. Supramolecularly controlled surface activity of an amphiphilic ligand. Application to aqueous biphasic hydroformylation of higher olefins. Catal. Sci. Technol. 1, 1347-1353 (2011).

22. Huang, J. H., Liu, Y. F. \& Wang, X. G. Selective adsorption of tannin from flavonoids by organically modified attapulgite clay. J. Hazard. Mater. 160, 382-387 (2008)

23. Lin, D. H. \& Xing, B. S. Tannic acid adsorption and its role for stabilizing carbon nanotube suspensions. Environ. Sci. Technol. 42, 5917-5923 (2008).

24. Han, X. X., Zhou, R. X., Yue, B. H. \& Zheng, X. M. Selective hydrogenation of cinnamaldehyde over $\mathrm{Pt} / \mathrm{ZrO}_{2}$ catalyst modified by $\mathrm{Cr}, \mathrm{Mn}, \mathrm{Fe}, \mathrm{Co}$ and Ni. Catal. Lett. 109, 157-161 (2006)

25. Ren, Y. P. et al. Influence of steric hindrance of organic ligand on the structure of keggin-based coordination polymer. Inorg. Chem. 45, 4016-4023 (2006).

26. Bilke, J. L. \& O'Brien, P. On the two-ligand catalytic asymmetric deprotonation of $\mathrm{N}$-Boc pyrrolidine: probing the effect of the stoichiometric ligand. J. Org. Chem. 73, 6452-6454 (2008)

27. Melean, L. G. et al. Biphasic hydrogenation of $\alpha, \beta$-unsaturated aldehydes with hydrosoluble rhodium and ruthenium complexes. Catal Lett. 141, 709-716 (2011).

28. Nakano, Y., Takeshita, K. \& Tsutsumi, T. Adsorption mechanism of hexavalent chromium by redox within condensed-tannin gel. Water Res. 35, 496-500 (2001).

29. Sun, X. Huang, X., Liao, X. P. \& Shi, B. Adsorptive removal of $\mathrm{Cu}$ (II) from aqueous solutions using collagen-tannin resin. J. Hazard. Mater. 186, 1058-1063 (2011).

\section{Acknowledgments}

The researches were financially supported by Starting Research Found from Sichuan Normal University and Foundation of Sichuan Educational Committee (13ZB0150).

\section{Author contributions}

H.M. and X.L. conceived the idea. H.M. carried out materials fabrication. H.M., H.Y. and J.C. performed materials characterizations. H.M. conducted the catalytic reactions. H.M. and X.L. drafted the manuscript. All authors analyzed and discussed the experimental results.

\section{Additional information}

Supplementary information accompanies this paper at http://www.nature.com/ scientificreports

Competing financial interests: The authors declare no competing financial interests How to cite this article: Mao, H., Yu, H., Chen, J. \& Liao, X.P. Biphasic catalysis using amphiphilic polyphenols-chelated noble metals as highly active and selective catalysts. Sci. Rep. 3, 2226; DOI:10.1038/srep02226 (2013).

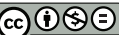

This work is licensed under a Creative Commons AttributionNonCommercial-NoDerivs 3.0 Unported license. To view a copy of this license, visit http://creativecommons.org/licenses/by-nc-nd/3.0 